\title{
La formación en comunicación en Tijuana: apuntes sobre un modelo de plan de estudios de la licenciatura en comunicación de la $U A B C$, Tijuana
}

\author{
David González Hernández* \\ Gerardo León Barrios** \\ Ángela Serrano Carrasco*** \\ Universidad Autónoma de Baja California, Unidad Tijuana
}

El presente artículo es una síntesis del documento original de la reestructuración del plan curricular en la licenciatura en comunicación en la UABC Tijuana, bajo el modelo por competencias. En él se expone, primeramente, un mapa de las dimensiones y contextos desde donde se hace dicha reestructuración. De manera central se exponen algunos aspectos metodológicos sobre los cuáles se diseñó y aplicó un estudio para diagnosticar la situación de la enseñanza de la comunicación en esta frontera. Finalmente, partiendo de los resultados de dicho diagnóstico, presentamos los ejes vectores sobre los que se fundamenta la nueva propuesta curricular para desarrollar las competencias de formación en el contexto fronterizo.

The present article is a synthesis of the curriculum plan in communication in the UABC Tijuana, under the professional competences educational model.

*Profesor de tiempo completo de la licenciatura en comunicación de la Universidad Autónoma de Baja California, Tijuana. Licenciado en comunicación por la Universidad Iberoamericana Tijuana, maestría en comunicación por el ITESO.

**Profesor de tiempo completo de la licenciatura en comunicación de la Universidad Autónoma de Baja California, Tijuana. Licenciado en Comunicación por la Universidad Iberoamericana Tijuana, maestría en comunicación por el ITESO.

***Profesora de tiempo completo de la licenciatura en comunicación de la Universidad Autónoma de Baja California, Tijuana. Licenciada en Comunicación Social y Periodismo, Universidad Externado de Colombia. Maestra en Estudios Latinoamericanos, FCPYS, UNAM. 
In it is exposed, primarily, a map of the dimensions and contexts that provide a clear scope of the construction logic of the curriculum. In a central way methodological aspects that helped the design of a diagnosis for what must be taught to communication students in the border are exposed. Finally, we are including the results of the diagnosis that served as a tool for the axes sequence both horizontally and vertically that aligned the new curriculum proposal to develop the competences of formation in the frontier context.

El presente escrito es una propuesta de modelo de plan de estudios de la licenciatura en comunicación de la Universidad Autónoma de Baja California (UABC), en la ciudad de Tijuana, y es a su vez una apuesta que se inscribe en el marco de la reflexión sobre la formación profesional de la comunicación en la región fronteriza y transfronteriza y en el mismo campo de la comunicación en México.

Por tanto, esta tarea académica implicó una profundización sobre varios aspectos del campo de la comunicación, sobre todo de la concepción de la comunicación ${ }^{1}$ para poder definir los referentes epistemológicos que orientaran el presente enfoque de plan de estudios. Pero, de manera fundamental, asumimos que uno de los referentes más importantes para el re-diseño curricular tendría que ser un diagnóstico que nos delineara los aspectos centrales sobre los cuales se desarrollarían las competencias profesionales, y a su vez, lograr tejer los hilos finos de toda una propuesta de formación profesional en nuestro contexto institucional y sociocultural.

Lo que presentamos aquí son los elementos básicos de lo que consiste la propuesta del plan de estudios de la licenciatura en comunicación, como resultado de la reestructuración del plan de estudios, realizada entre 2004 y 2005 , que entrará en vigor en agosto de 2006.

\section{Dimensiones Y CONTEXTOS.}

En ejercicio de colocar el planteamiento del re-diseño curricular sobre los referentes que le dan la vida actual tanto a la formación de profesionales de la comu-

${ }^{1}$ Como resultado de una discusión orientada por asesorías y seminarios para el diseño de esta propuesta, pensamos a la comunicación como una práctica social que permite el encuentro e intercambio de sentidos y significados - conceptos, ideas, una visión de la vida y del mundo- que ocurre permanentemente entre dos o más personas; siempre y cuando esta práctica posibilite la interacción humana dentro de un sistema sociocultural específico (Torrico, 2004).

430 • David González Hernández, Gerardo León Barrios, Ángela Serrano Carrasco 
nicación como a la vida profesional de éstos, tomamos como referencia cuatro ámbitos que se articulan en el rediseño curricular de manera sistémica:

\section{Contexto sociocultural}

Este identifica las particularidades socioculturales que representa vivir en la frontera. En este ámbito encontramos que la relación transfronteriza, aunque limitada, ha posibilitado la construcción de unidades socio-ecológicas, económicas, sociales, culturales y familiares de carácter regional.

La vecindad geográfica ha producido un fenómeno de relaciones sociales que se pueden comprender como un conjunto de interacciones entre actores de distintas naciones, niveles de desarrollo económico, tradiciones y valores culturales y con diferente grado de poder $^{2}$. Debido a este importante factor, la internacionalidad de las interacciones fronterizas o transfronterizas entre mexicanos y estadounidenses, ha ocurrido en un contexto estructural caracterizado por la desigualdad o asimetría de poder entre los países, tanto en el orden económico como político, teniendo como consecuencia creaciones ideológico-políticas por ambas partes. ${ }^{3}$

\section{Contexto de formación y desarrollo socio-profesional}

Refiere a la realidad de la educación superior, la formación del profesional de la comunicación y su desempeño en el mercado laboral. En el ámbito nacional, los egresados de comunicación en 1991, sumaban un total de 3,839, con 2,421 provenientes de las instituciones públicas (63\%) y 1,418 en privadas. Para el 2000, el número de egresados alcanzaban los 7,033 (un incremento del 183\%). De esa cantidad, 3,402 egresaron de instituciones públicas y 3,631, de escuelas privadas ${ }^{4}$

En Baja California hay una oferta excedente del mercado laboral en educación, humanidades y en ciencias sociales y administrativas, áreas de estudio donde se ubican los egresados de comunicación. De acuerdo al informe de ANUIES, en la proyección de profesionistas ocupados por áreas de estudio en la región noroeste destaca en primer lugar las ciencias sociales y administrativas

\footnotetext{
${ }^{2}$ Bustamante, Jorge (1989): Frontera México-Estados Unidos; reflexiones para un marco teórico, Frontera Norte. Volumen I, No. 1 (enero-junio).

${ }^{3}$ INEGI. XII Censo General de Población y Vivienda, 2000.

${ }^{4}$ Asociación Nacional de Universidades e Instituciones de Educación Superior (ANUIES). Mercado Laboral de Profesionistas en México: Diagnóstico y prospectiva al año 2010.
}

\section{La formación en comunicación en Tijuana: apuntes sobre un modelo de plan de estudios $\bullet 431$ de la licenciatura en comunicación de la UABC, Tijuana}


(con más del $40 \%$ del total), y en tercer lugar la educación y humanidades (con casi el 14\%), cuyo promedio de remuneración en pesos en Baja California es de 8,937 pesos (ciencias sociales y administrativas) y 6,159 pesos (educación y humanidades). En estos porcentajes, domina el egreso de las instituciones públicas en Baja California (más del $85 \%$ en ambos casos).

\section{Dimensión disciplinar}

Esta nos permitió identificar cierta debilidad epistemológica en el de la comunicación como objeto de estudio (Fuentes, 1991; Galindo, 2003; Torrico, 2004), lo cual impacta en la formación profesional en tanto que la enseñanza, así como lo difuso del objeto del conocimiento, se caracteriza por un compendio de saberes desarticulados y muchas habilidades en el uso de tecnologías a lo largo de la formación del profesional de la comunicación.

Sabemos que hay dos elementos importantes que intervienen en la configuración de un campo académico: la producción de conocimiento y la institucionalización de la enseñanza. En el caso mexicano, gran parte del insumo teórico-metodológico sobre los aspectos de comunicación provienen de la investigación, la cual está lejos de realizarse conforme a una estructura estable. Se trata de un área académica en continua reformulación, tanto de lo que estudia como las formas de hacerlo, así como su enseñanza y sus programas institucionales. ${ }^{5}$ La enseñanza de la comunicación encuentra su asidero en los medios masivos, especialmente en la formación para la producción de mensajes.

\section{Dimensión de politicas institucionales de la UABC}

Ángulo que definen el marco de referencia orgánico donde se inscribirá el nuevo plan de estudios. Como retos institucionales, algunos de ellos trazados por el Plan de Desarrollo Institucional, tenemos el pensar la formación de profesionales de la comunicación a partir de una estrecha vinculación con el entorno social, mediante estrategias de vinculación con la comunidad a partir

${ }^{5}$ Un aspecto que argumenta lo que venimos diciendo es la baja producción de libros sobre comunicación. Esto plantea muchas problemáticas, en especial en la enseñanza de la comunicación en el contexto mexicano. De esta manera, el insumo teórico que permite la identificación y reflexión de procesos de comunicación contemporáneos en sus distintas dimensiones, resulta incipiente para la formación en las carreras de comunicación en México, y lo que emerge entonces, es la disociación entre teoría y práctica, generando por tanto una distancia fuertemente marcada entre la enseńanza de la comunicación y el campo profesional, entre universidad y profesión. En Fuentes, R. (2003). La investigación académica sobre comunicación en México. Guadalajara, México: ITESO.

432 • David González Hernández, Gerardo León Barrios, Ángela Serrano Carrasco 
del servicio social comunitario, el servicio social profesional y las prácticas profesionales. La formación profesional del comunicólogo debe construirse a partir de competencias básicas y específicas y debe caracterizarse por la apertura de dinámicas centradas en la resolución de problemas sociales. La formación profesional deberá apoyarse por dinámicas de movilidad estudiantil a corto plazo, que permitan un mayor intercambio con la DES de Ciencias Sociales y Humanidades, con otras DES de la UABC, así como con otras instituciones académicas regionales, nacionales e internacionales a mediano y largo plazo.

Por lo tanto, los argumentos fundamentales sobre los cuales planteamos el rediseño curricular tienen tres ejes:

1. Una estrecha correspondencia entre el proyecto universitario de la UABC y las demandas sociales del entorno fronterizo. Ante esto, es de suma importancia reconocer la condición histórico social por la que atraviesa nuestra sociedad de inicios de siglo XXI, considerando el carácter global de las interacciones económicas, políticas, pero sobre todo sociales que afectan tanto lo local como lo regional.

2. La urgencia de superar la "escisión teoría-práctica", articulando la generación de conocimiento en comunicación con la práctica profesional misma de la comunicación, y ver que en ello está el centro de atención de este nuevo proyecto académico. Para ello, la teoría dentro del nuevo programa de estudios ocupa un lugar central en la medida que provea de herramientas conceptuales y metodológicas la práctica profesional más allá del acumulamiento de conocimiento.

3. La necesidad de pasar del "mediacentrismo" en que se ha desarrollado por varias décadas la enseñanza de la comunicación en México, y trabajar desde una perspectiva más amplia que forme más en la capacidad de intervención de lo comunicacional, diversificando los enfoques profesionales hacia áreas emergentes, sin olvidar que la producción de discursos constituye el área tradicional por excelencia de la formación de comunicadores en el ámbito latinoamericano. ${ }^{6}$

INVESTIGACIÓN PARA DIAGNOSTICAR EL PLAN DE ESTUDIOS I997-I

Orientados por las argumentaciones anteriores, asumimos la tarea de realizar una investigación y así tener un diagnóstico (Prieto, 1988) sobre situación el

${ }^{6}$ Seminario "Competencias en Comunicación" con el Dr. Raúl Fuentes Navarro. Tijuana, 6 y 7 de septiembre de 2004, UABC, Tijuana.

La formación en comunicación en Tijuana: apuntes sobre un modelo de plan de estudios $\bullet 433$ de la licenciatura en comunicación de la UABC, Tijuana 
plan de estudios 1997-1 que nos generara información de primera mano sobre la compleja relación entre el funcionamiento de la currícula de la Licenciatura en Comunicación y la vida profesional de un egresado en el campo ocupacional en la ciudad de Tijuana.

Metodológicamente nos vimos en la necesidad de definir dos dimensiones para nuestro estudio: una interna, referida al aspecto institucional de la enseñanza, el cual nos permitió conocer la operación del plan de estudios vigente con el fin de evaluar sus potencialidades y debilidades en la formación de profesionales. Otra externa, relacionada con el aspecto socio-profesional, que nos aproxima a los escenarios, condiciones y necesidades en las que se encuentra actualmente la vida profesional de un comunicólogo en la región.

Los sujetos de estudio para cada dimensión fueron: alumnos y profesores para la interna; egresados y empleadores, para la externa. Desde la una estrategia multi-método, aplicamos diferentes métodos y técnicas pertinentes para cada uno de los grupos de sujetos de estudio, y generar información de carácter empírico de esta forma: con alumnos, una sesión de grupo; con profesores, un taller a futuro; con egresados, una encuesta exploratoria y por último, con empleadores, una entrevista semi-estructurada.

\begin{tabular}{lll} 
Dimensión & SujeTOS DE ESTUdiO & PAQUETE TECNOLÓGICO \\
\hline a) Interna & Alumnos & Sesión de grupo \\
& Profesores & Taller a futuro \\
\hline b) Externa & Egresados & Encuesta exploratoria \\
& Empleadores & Entrevista semi-estruturada
\end{tabular}

El programa metodológico de este diagnóstico se desarrolló bajo tres ejes que se articularon de manera transversal en la construcción de los datos: 1) la formación académica que reciben los estudiantes de Comunicación en la UABC; 2) la descripción del campo profesional y laboral del comunicólogo, incluyendo las condiciones laborales características de la ciudad de Tijuana; y 3) la relación entre la formación académica y el desempeño profesional.

\section{Resultados: DE LOS DATOS A LOS OBSERVABLES}

De las dos dimensiones y, a su vez, de las cuatro unidades de análisis, nos permitieron generar información que nos llevó a un diagnóstico sobre la situación formativa y profesional del comunicólogo egresado de la UABC Tijuana sobre cuatro elementos constitutivos de la formación-función del profesional de la comunicación en Tijuana formado en la UABC. 
a) De la enseñanza de la comunicación en estudiantes de la licenciatura del Plan actual 1997-1. El análisis de las percepciones sobre estudiantes nos indicó que en el esquema curricular y pedagógico a implementarse, se repensar que la enseñaza en comunicación debe: a) revertir una desarticulación entre materias, y, a su vez, entre los objetos formativos y perfiles; b) la escisión entre la formación teórica y la formación práctica, para facilitar la integración de conocimientos en función del (los) perfil(es) profesional(es); c) el diseño del nuevo plan de estudio debería buscar tener un enfoque integrado tanto en términos de contenido como de operatividad, en tanto se enseñan saberes bajo modelos pedagógicos distantes de la realidad sociocultural de los estudiantes; d) es urgente en la formación profesional tener espacios curriculares en donde el estudiante haga visible los conocimientos, habilidades y actitudes aprendidas en las diferentes etapas formativas; e) por otra parte, el tema pendiente y en construcción, es la redefinición de la labor social y campo profesional del comunicólogo desde el nivel de formación académica de la licenciatura, para lo cual, los procesos de evaluación del nuevo plan de estudios nos permitirán tener espacios vinculación y monitoreo de estudiantes y egresados dentro del campo laboral.

b) Descripción del campo profesional y laboral del comunicólogo en Tijuana. Debido a la relativa creación de la carrera de comunicación en la Universidad Autónoma de Baja California (1991) el 94.5\% de nuestros egresados es menor de 35 años, lo que nos dice que la experiencia profesional es todavía joven y en proceso de consolidación en el campo laboral en la región. El 82.4\% no están titulados y por lo tanto, muchos de ellos ejercen en el área en un nivel técnico, o bien, poco vinculado con el oficio de la comunicación. En términos de conocimientos profesionales, los empleadores perciben a nuestros egresados más capacitados para la producción de mensajes en medios de comunicación masiva que los egresados de otras universidades de la ciudad. Sin embargo, los empleadores identifican en los egresados una ausencia de información sobre la realidad social, política, económica y cultural; lo cual evidencia la falta de hábito en la búsqueda de información general y el pensamiento analítico. Esta situación conlleva a que los comunicadores egresados de la UABC carezcan de un sentido práctico necesario para la resolución de tareas diarias concernientes a la labor profesional del comunicólogo. En nuestro estudio identificamos como dato relevante, que, por parte de los empleadores, no hay una claridad respecto a los aportes que un profesional de la comunicación le traería a sus respectivas organizaciones, pero, tampoco el profesional de la comunicación lo sabe. Se percibe cierta indefinición del oficio que corresponde al profesional de la comu-

La formación en comunicación en Tijuana: apuntes sobre un modelo de plan de estudios $\bullet 435$ de la licenciatura en comunicación de la UABC, Tijuana 
nicación y los beneficios de su intervención, con el agravante de que, tal como lo señalamos arriba, el comunicólogo egresado tampoco lo tiene claro.

El reto del nuevo plan consiste entonces, en propiciar la habilidad en la reflexión y análisis sobre el contexto sociocultural e histórico en el que se ubican las dinámicas contemporáneas de la comunicación (lo global y lo local). La tarea de la universidad consiste en diseñar estrategias de acercamiento al sector laboral con el fin de posicionar la función del comunicólogo y los objetos socioprofesionales de la comunicación que se están formando en la UABC para la región.

c) Campo profesional del comunicólogo en Tijuana. Para definir este aspecto, retomamos la experiencia profesional de nuestro egresado en tres momentos: antes de su egreso, después del egreso y en su trabajo actual. Las áreas que fueron una constante en los tres momentos de la historia laboral fueron 1) el tradicional campo profesional de los medios masivos de comunicación (radio, televisión, prensa y cine) y 2) la promoción y la mercadotecnia.

Las áreas identificadas como emergentes en la región son: la comunicación organizacional y las nuevas tecnologías de comunicación.

La oferta laboral en las áreas tradicionales (medios de comunicación) es reducida en relación con la cantidad de egresados de las carreras de comunicación en la ciudad. Debido a esto, una opción de inserción en el mercado laboral es incursionar en las áreas de mercadotecnia y promoción, las cuales, en muchas ocasiones, se traducen en actividades de ventas.

El giro de las instituciones donde laboran actualmente es diverso, sobresaliendo el sector privado (27.17\%), seguido por el gubernamental (19.5\%) y los servicios independientes (17.39\%).

d) Relación entre la formación académica y el campo profesional. Desde la experiencia profesional de los egresados, los conocimientos más requeridos en el campo son: el dominio en la producción de medios de comunicación, el manejo de programas (software) y la comunicación organizacional; seguido por las relaciones públicas y las nuevas tecnologías en comunicación. Las habilidades esenciales para el desempeño profesional, destacan: resolver problemas, manejar información, estructurar ideas, la expresión o comunicación oral, la creatividad, saber analizar, buscar información y argumentar propuestas.

En síntesis y a partir de los resultados de las cuatro dimensiones de nuestro diagnóstico, el diseño de la propuesta del plan de estudios contempló la formación del comunicólogo en la capacidad de intervención en tres problemáticas de su realidad, aplicando saberes teórico-prácticos de la comunicación, esto es, un interventor social que sepa identificar en tres niveles las problemáticas propias

436 • David González Hernández, Gerardo León Barrios, Ángela Serrano Carrasco 
en los procesos de interacción social aplicando conocimientos y habilidades para diseñar estrategias que ofrezcan una solución y evaluar su impacto:

1. Dificultad para identificar los procesos de comunicación existentes en el contexto social, cultural, político, económico e histórico, así como las problemáticas comunicacionales asociadas a dichos procesos.

2. Al comunicólogo se le dificulta proponer soluciones a problemas comunicacionales debido a la falta de articulación de sus recursos (conocimientos, habilidades y actitudes) para enfrentarse a las transformaciones del campo profesional.

3. Al comunicólogo se le dificulta resolver problemas de comunicación lo cual lo hace participar limitadamente en el ámbito profesional.

Del DiAgNóstico a los RESUltados:

LAS TRES PROBLEMÁTICAS PARA EL DISEÑO DE COMPETENCIAS

La propuesta del plan de estudio se diseńó bajo el modelo de competencias en correspondencia con la filosofía y el modelo educativo de la Universidad Autónoma de Baja California. El modelo de competencias establece la adquisición y desarrollo de capacidades tanto en la pericia del "saber hacer" como en la interacción y comportamiento social.

De esta manera, además de plantear nuevas formas de enseñanza-aprendizaje en la licenciatura de comunicación, se tomaron en cuenta aspectos como la estructuración de conocimientos, habilidades y actitudes para el diseño curricular que permitieran una mayor articulación entre las asignaturas en función de competencias generales a desarrollar en el alumno. Cabe recordar que dichas competencias generales emergieron de los análisis transversales, y considerando los aspectos contextuales, disciplinares, socio-profesionales e institucionales que inciden en la vida profesional del comunicólogo.

De ahí las tres competencias generales que propone este plan de estudios en los futuros estudiantes de comunicación.

- Identificar procesos y problemáticas de comunicación articulando información de la realidad social, política, cultural, económica e histórica en distintos ámbitos y niveles del entorno, utilizando diversas herramientas teórico-metodológicas propias de las ciencias sociales para su análisis.

De acuerdo a las condiciones del campo académico de la comunicación en México y en el mundo, la dificultad para identificar los procesos de la comunicación resulta la más común para los profesionistas de la comunicación: el

La formación en comunicación en Tijuana: apuntes sobre un modelo de plan de estudios $\bullet 437$ de la licenciatura en comunicación de la UABC, Tijuana 
hecho comunicativo se identifica en las interacciones y prácticas humanas, y en las mediaciones tecnológicas (medios masivos de comunicación) que ponen en circulación una inmensa cantidad de mensajes en la sociedad, lo que presenta muchas problemáticas de entendimiento teórico y práctico en varias dimensiones.

- Proponer estrategias de solución a problemas de comunicación, articulando los conocimientos, habilidades y actitudes propias de la disciplina, de manera innovadora que impulsen el desarrollo e impacto social de la organización donde participa.

Este aspecto representa una problemática que identifica una de las carencias profesionales que enfrenta el egresado en comunicación, que tiene que ver con la capacidad de proponer estrategias de solución a problemas de comunicación y que tiene también su antecedente en la manera dispersa de conceptualizar la comunicación en el mismo campo académico de la comunicación. Por un lado, sabe cuestiones teóricas básicas sobre comunicación, siendo uno de sus rasgos la capacidad analítica y lógica (34.1\%) y la habilidad para la búsqueda de información (31.9\%) y otros elementos humanísticos; pero del otro lado, ha desarrollado habilidades para el uso de tecnología en medios.

El profesional de la comunicación experimenta la escisión entre teoría y la práctica, pues no ha logrado incorporar a la visión de su disciplina, saberes que -articulados- le permiten ser competente para diseñar o proponer estrategias de solución (en el diagnóstico este elemento representa el 76.9\% de las necesidades del mercado laboral).

Lo anterior revela una carencia del plan de estudios actual, en tanto no hay una articulación explícita, constante y clara entre las asignaturas de tipo teórico con las asignaturas de tipo práctico, así como de las actividades de aprendizaje y las perspectivas de los maestros acerca del enfoque profesional del estudiante de comunicación que genere en los alumnos la competencia de argumentar propuestas.

- Resolver problemas de comunicación en diferentes ámbitos profesionales, integrándose a grupos multidisciplinarios utilizando los recursos teóricometodológicos propios de las ciencias sociales, a fin de contribuir al desarrollo social considerando las dinámicas del entorno transfronterizo.

Siguiendo los fundamentos de las competencias a desarrollar, y en relación también a nuestro diagnóstico, la tarea profesional de llevar a cabo propuestas --

438 - David González Hernández, Gerardo León Barrios, Ángela Serrano Carrasco 
una vez que se genera la competencia para ubicar y contextualizar problemáticas comunicacionales, así como también tener la capacidad de diseñar propuestas de solución- la actividad de ejecutar estas propuestas aparece como uno de los grandes retos que el profesional de la comunicación debe enfrentar en cualquier ámbito laboral, y adquiere mayor fundamento al incorporar el resultado del diagnóstico donde, en voz de los egresados, la habilidad para aplicar estrategias de solución está representada con un $74.7 \%$ como una cualidad esencial del nuevo comunicólogo en la región.

La manera en que visualizamos cómo el profesional de la comunicación enfrenta el escenario profesional es la siguiente:

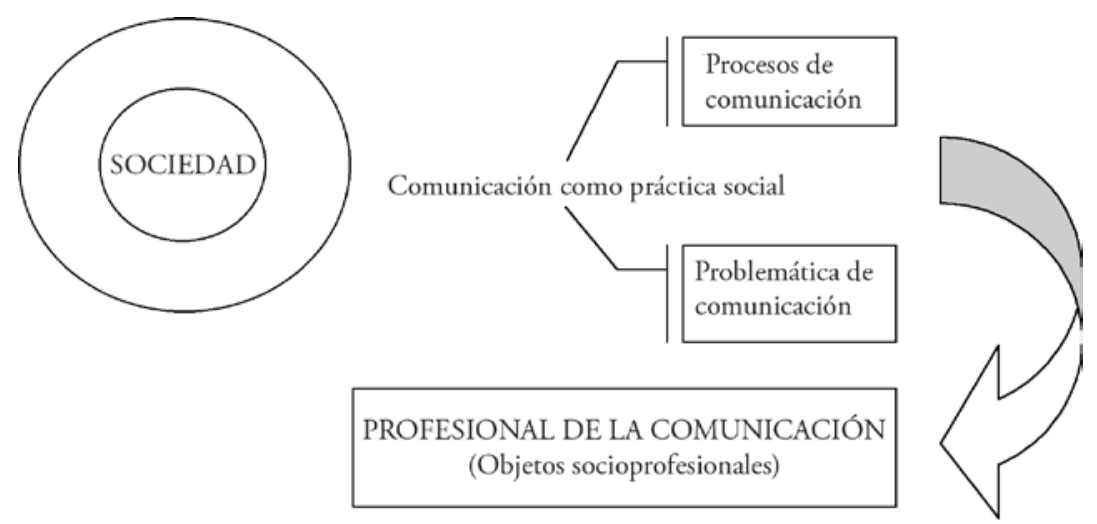

NÚCLEOS FORMATIVOS

\begin{tabular}{|c|c|c|c|}
\hline & $\begin{array}{l}\text { UBICACIÓN } \\
\text { CONTEXTUALIZACIÓN }\end{array}$ & $\begin{array}{l}\text { Diseño de } \\
\text { PROPUESTA }\end{array}$ & $\begin{array}{l}\text { APLICACIÓN } \\
\text { Y EVALUACIÓN }\end{array}$ \\
\hline $\begin{array}{l}\text { COMPETENCIA } \\
\text { FORMATIVA }\end{array}$ & $\begin{array}{l}\text { Identificar procesos } \\
\text { y problemáticas de } \\
\text { comunicación en } \\
\text { distintos ámbitos del } \\
\text { contexto en el que } \\
\text { se ubica. }\end{array}$ & $\begin{array}{l}\text { Proponer o mejo- } \\
\text { rar problemas de } \\
\text { comunicación en di- } \\
\text { ferentes dimensiones } \\
\text { de los procesos de } \\
\text { comunicación }\end{array}$ & $\begin{array}{l}\text { Implementar estrate- } \\
\text { gias de comunicación, } \\
\text { integrándose a grupos } \\
\text { multidisiciplinarios; } \\
\text { y evaluar el impacto } \\
\text { de la estrategia en el } \\
\text { ámbitos social donde } \\
\text { se ha aplicado }\end{array}$ \\
\hline
\end{tabular}

Lo anterior podrá ser puesto en práctica mediante los dos perfiles que bien pueden combinarse entre sí: el estratega de la comunicación y el productor de mensajes.

La formación en comunicación en Tijuana: apuntes sobre un modelo de plan de estudios 439 de la licenciatura en comunicación de la UABC, Tijuana 
El estratega de la comunicación se define como un planificador de acciones comunicativas, capaz de identificar problemáticas en los procesos de comunicación intersubjetivos y más mediáticos. Puede definir y llevar a cabo acciones para el mejoramiento de tales procesos en función de objetivos organizacionales, ya que posee las herramientas teórico metodológicas para intervenir. Podrá laborar como independiente en consultarías de comunicación, o bien, inserto en organizaciones e instituciones de diversa índole.

El productor de mensajes, es capaz de analizar diferentes tipos de productos audiovisuales y puede combinar criticidad y creatividad en la elaboración de unos nuevos, ya que posee los conocimientos técnicos necesarios para ello. Podrá laborar de manera independiente o inserto en una organización o institución, realizando tareas de periodista o en productoras de medios audiovisuales. Lo anterior podrá ser puesto en práctica mediante dos oficios que bien pueden combinarse entre sí: el estratega de la comunicación y el productor de mensajes.

El estratega de la comunicación se define como un planificador de acciones, situaciones o procesos de comunicación, capaz de identificar problemáticas en los procesos de comunicación intersubjetivos y mass mediáticos. Puede definir y llevar a cabo acciones para el mejoramiento de tales procesos en función de objetivos organizacionales, ya que posee las herramientas teórico metodológicas para intervenir. Podrá laborar como independiente en consultorías de comunicación, o bien, inserto en organizaciones e instituciones de diversa índole.

El esbozo de la propuesta de plan de estudios aquí presentada puede parecer no novedosa en algunos aspectos dentro del campo de la comunicación. Si bien desde los años ochenta ya aparecía como necesario replantear el rumbo de la enseñanza en comunicación en función del proyecto social del profesional de la comunicación (Fuentes, 1991; Galindo, 1995; Prieto, 1988), a lo que nos enfrentó este ejercicio de diseño fue ver que las dinámicas del campo han tenido un efecto retardado en cuanto a los principios con los cuales se "enseña" comunicación en esta parte del país, y ante ello, nos tocó comprender que el carácter global de muchos de los aspectos socioculturales de la región estaban siendo transformados profundamente. El modelo que se propone, entonces, intenta poner en sintonía algunos principios educativos sobre comunicación en este contexto de profundos cambios y acelerados procesos de nuestra vida contemporánea en la frontera. 


\section{BiBLIOGRAFÍA}

Alonso, L. E. (1994). Sujeto y discurso: El lugar de la entrevista abierta en las prácticas de la sociología cualitativa, en Juan Manuel Delgado y Juan Gutiérrez (coords.); Métodos y técnicas cualitativas de investigación en ciencias sociales, Síntesis, Madrid.

Anderson y De la Rosa (1991). Familias pobres de la frontera. Estrategias de sobrevivencia en Estudios sobre culturas contemporáneas. Número 11. Volumen IV. Universidad de Colima, Colima.

ANUIES (Asociación Nacional de Universidades e Instituciones de Educación Superior) Mercados laborales de profesionistas en México. Diagnóstico y prospectiva al 2010. México, Recuperado en enero del 2005 de: http://www. anuies.mx/anuies.

Fuentes, R., (2001). Comunicación, utopia y aprendizaje: propuestas de interpretación y acción : 1980-1996. México: ITESO Guadalajara.

(2000). Perspectivas socioculturales postdisciplinarias en lainvestigación de la comunicación. En Orozco, Guillermo, Lo viejo y lo nuevo. Investigar la comunicación en el siglo XXI. Ediciones de la Torre. Argentina

(1991). Diseño Curricular para las Escuelas de Comunicación . México: Trillas.

Galindo, J. (2003). Sobre comunicología y comunicometodología. Primera guía de apuntes sobre horizontes de lo posible. Artículo en línea. Recuperado en septiembre de 2005 de: http://www.geocities.com/arewara/arewara

Galindo, J. y Luna, C., (1995). Campo académico de la comunicación: hacia una reconstrucción reflexiva. México: ITESO-CONACULTA

Ibáñez, J. (1979). Más allá de la sociología. El grupo de discusión: técnica y crítica. Madrid: Siglo XXI.

López, H. (1998). La metodología de la encuesta, en Galindo Cáceres, Luis, Técnicas de investigación en sociedad, cultura y comunicación, Addison Wesley Logman, México.

Mier, L. J. (1987). En busca de un proyecto integral en la formación de comunicadores, en Diálogos de la Comunicación, No. 18, FELAFACS, Perú.

Reguillo, R. (1998). De la pasión metodológica o de la (paradójica) posibilidad de la investigación.. Mejía, Rebeca y Sandoval. En Tras las vetas de la investigación cualitativa. México: ITESO.

Prieto, D. (1988). Diseño curricular para escuelas de comunicación. Quito, Ecuador: CIESPAL.

Torrico, E. (2004). Abordajes y periodos de la teoría de la comunicación. Buenos Aires: Norma.

La formación en comunicación en Tijuana: apuntes sobre un modelo de plan de estudios 441 de la licenciatura en comunicación de la UABC, Tijuana 